P145 (continued)

compares 2nd and 3rd grade students' consumption of various subtypes of vegetables served as part of hot lunch.

Design, Setting, Participants, and Intervention: Second and 3rd grade students from 14 NYC public elementary schools were observed on at least 3 days during lunch. On observation days, 3-6 researchers observed 4 random students each, using the System of Observational Cafeteria Assessment of Foods Eaten (SOCAFE).

Outcome Measures and Analysis: Vegetable selection was recorded, as was amount consumed $(0 \%, 10 \% / b i t e$, $25 \%, 50 \%, 75 \%, 100 \%,>100 \%)$. Vegetables served were classified into 6 subtypes. One-way ANOVA and post-hoc Tukey HSD tested for differences in mean intake across subtypes.

Results: Students' consumption of vegetables was: white potato $(49 \%, \mathrm{SD}=51 \%)$; other starchy: corn, plantains $(28 \%, S D=44 \%)$; dark green: broccoli, garden salad, kale $(12 \%, \mathrm{SD}=30 \%)$; red/orange: tomatoes, sweet potatoes, carrots $(8 \%, \mathrm{SD}=22 \%)$; beans: black beans, chickpeas, $(1 \%, \mathrm{SD}=4 \%)$; and other: cucumber, green beans, mixed vegetables in egg roll $(10 \%, \mathrm{SD}=24 \%)$. Consumption of white potato and other starchy vegetables were significantly higher than all other subtypes, all at $\mathrm{p}<0.001$, with potato also higher than other starchy, $\mathrm{p}<0.01$. Dark green vegetable consumption was statistically higher than beans, $\mathrm{p}<0.05$.

Conclusions and Implications: This study confirms what others have found, that when white potatoes are served, students eat more vegetables. Students also eat more other starchy vegetables. yet, consumption of all other vegetables were low. Nutrition education that include motivational activities, tasting and cooking can be used to encourage students to eat a wider variety vegetables, specifically those less frequently consumed at school lunch.

Funding: Wellness In The Schools

\section{P146 The Effect of School Lunch Interventions and Policy Changes on Student Food Behavior 2006-2016: A Systematic Review}

Jennifer Mansfield, BS, jmansfi@purdue.edu, Purdue University, 700 West State Street, West Lafayette, IN 47907; D. Savaiano, PhD

Objective: Childhood obesity is a global epidemic. One approach addressing childhood obesity is through federally subsidized school meals programs. The U.S. National School Lunch Program mandates school nutrition standards aligned with the Dietary Guidelines for Americans. As students consume a significant proportion of their total intake during the school day, increasing access to healthy foods at school is proposed to improve student dietary outcomes. The purpose of this review is to assess how school lunch interventions and policy changes in the last ten years (namely the 2006-2007 Wellness Policies and the Healthy, Hunger-Free Kids Act of 2010) affected student food behavior.
Design, Setting, and Participations: PubMed, Web of Science, and Science Direct were searched for primary research studies evaluating student food behavior in the U.S. school lunch setting. Only policy evaluations and interventions implemented during specified policy time periods were included. A total of twenty-nine studies meeting inclusion criteria were identified and reviewed.

Outcome Measures and Analysis: Plate waste, intake, selection, and purchasing patterns.

Results: Seventeen studies reported improved food behavior changes (increased selection, intake, and sales of healthy foods, and decreased plate waste). Twelve studies reported negative food behavior outcomes (decreased selection, intake, and sales of healthy foods, and increased plate waste).

Conclusions and Implications: The majority of studies indicated that increasing access to healthy foods during school lunch improved students' dietary outcomes. However, these findings are not consistent across the literature. Study Design, adaptation period, sample size, food culture, quality of foods, and policy implementation challenges contribute to the success/failure of school lunch. Ongoing evaluation of these programs is warranted.

Funding: None

\section{P147 Designing and Evaluating a Training Protocol for Visual Estimation of Fruits and Vegetable Intake Among K-2nd Grade Students}

Natalie Masis, MS, masis2@illinois.edu, University of Illinois, 238 Bevier Hall, 905 South Goodwin Avenue, Urbana, IL 61801; K. Chapman-Novakofski, PhD, RD, LDN; $J$. McCaffrey, $P h D, R D$, University of Illinois Extension; S. Johnson, PhD, University of Colorado at Denver

Objective: To design a replicable training protocol for visual estimation of fruit and vegetable (FV) intake of $\mathrm{K}-2$ nd grade students through digital photography of lunch trays that results in trainees recording reliable data for food served and food eaten.

Design, Setting, Participants, and Intervention: Protocol development through literature and researcher input was followed by 3 trainings of 3 trainees and a practice session at a local elementary school. Data were then collected at 2 elementary schools for K-2nd graders' lunches. School 1 included nutrition education and salad bar; school 2 was control school.

Outcome Measures and Analysis: Intraclass correlation coefficients (ICC) for trainings and lunchroom data collection.

Results: By training 3, ICC was substantial for amount of FV served (0.86, $\mathrm{p}<.01)$; percentage FV consumed (0.954, $\mathrm{p}<.01)$, amount $\mathrm{F}$ served $(0.97, \mathrm{p}<.01)$; amount $\mathrm{V}$ served $(0.81, \mathrm{p}<.01)$; percentage $\mathrm{V}$ consumed $(0.98, \mathrm{p}<.01)$. In-school estimates for ICCs were all significant for amounts served at 1 school, and amount FV consumed at both schools. Mean percentages consumed differed between the 2 schools (fruit consumed: $76.9 \%+38 \%$ school 
P147 (continued)

1 vs $42.2 \%+40 \%$ school 2 ; vegetable consumed: $67 \%+$ $44 \%$ school 1 vs $18 \%+32 \%$ school 2 ).

Conclusions and Implications: The protocol resulted in reliable estimation of FV intake using digital photography. Being able to accurately estimate FV intake will benefit intervention development and evaluation.

Funding: University of Illinois Extension

\section{P148 Nutrition Education in the Classroom Utilizing MyPlate Improves Children's Knowledge of Food Groups and Preferences in Kindergarten}

Tomoko Osera,PhD, tomokocup12@yahoo.co.jp, Hygiene and Preventive Medicine, Graduate School of Life Science, Kobe Women's University, 4 Chrome-7-2 Minatojima Nakamachi, Chuo Ward, Kobe, Hyogo Prefecture 650-0046, Japan; S. Tsutie, MS; Y. Segawa, MS; M. Kobayashi, MS, Takakuradai Kindergarten at Kobe Women's University; N. Kurihara, PhD, Kobe Women's University

Objective: To examine the effects of a nutrition education program using MyPlate on food group knowledge and food preference in kindergarten age children.

Target Audience: A total of 80 children (mean $5.1 \pm 0.2$ ages) attending kindergarten participated in the study.

Theory, Prior Research, Rationale: The provision of knowledge does not necessarily lead to a healthy lifestyle. Knowledge is the first step to change behavior. Teachers and nutritionists provided information about food groups to children using MyPlate.

Description: A quasi-experimental pre- post-test design was used to examine the effect of the nutrition education program. Both the intervention group $(n=55)$ and comparison group $(n=25)$ received basic nutrition education from their teachers that included gardening and cooking at least once a month. Children in the intervention group received an additional 10 minutes of nutrition education with MyPlate during lunchtime, which was held 4 times/week for 16 weeks.

Evaluation: The survey assessing children's food preferences was completed by their parents and the survey assessing children's knowledge about food groups was completed by the children. A measurement scale was used for dichotomous responses. Based on food preference, the number of disliked foods was significantly less in the intervention group compared with the comparison group $(\mathrm{p}<0.05)$. The improvement in knowledge about food groups in the intervention group was significantly higher than the comparison group $(\mathrm{p}<0.001)$.

Conclusions and Implications: Nutrition education involving the MyPlate program is suggested to increase knowledge about food groups and improve food preference in kindergarten age children. This program may be useful for changing children's food preferences in kindergarten age children.

Funding: None
P149 Knowledge, Attitudes and Practices of Nutrition and Physical Activity in Children of Primary Schools in Chiapas, Yucatan and Quintana Roo, Mexico

Mariana Perez, MA, mariana.perez@savethechildren.org, Save the Children, Francisco Sosa No. 30 Col. Villa, Coyoacan Del. Coyoacan, Mexico, DF C.P 04000; L. Saltijeral, $M A$

Objective: To identify the knowledge, attitudes and practices of nutrition and physical activity in children of primary schools in Chiapas, Quintana Roo and Yucatan, Mexico.

Design, Setting, and Participants: The diagnosis was conducted in October 2015 in 36 primary schools (19 in Chiapas, 4 in Quintana Roo and 13 in Yucatan). A Knowledge, Attitudes and Practices survey (KAP) was applied to a statically significant randomized sample of 1292 students from grades 4 th through 6 th. The data analysis was descriptive.

Results: Forty-nine percent of the respondents were boys and $51 \%$ girls. The analysis shows, $69 \%$ of the children reported plain water consumption at school the day before the survey, and that $15 \%$ had drank three or more sugarsweetened beverages; $70 \%$ had consumed vegetables and $66 \%$ had consumed at least one fruit. However, 43\% consumed fried foods and 51\% sweets or pastries, the day prior to the evaluation. Concerning the practice of physical activity (PA), 97\% of respondents considered it as favorable to keeping a healthy weight and 95\% said that being overweight affects health; however, only $28 \%$ performed at least 30 minutes of PA three times a week.

Conclusions and Implications: It is necessary to make greater efforts to promote the practice of PA in school. Educational strategies focused on the modification of eating habits and PA for health, should build on knowledge and attitudes prevalent in the school population, in order to ensure the success of educational interventions. Funding: FEMSA

\section{P150 National Competitive Foods Standards in Rural Virginia Middle Schools: Smarter Snacking or Compliance Lacking?}

Georgianna Mann, PhD, gmann89@vt.edu, Virginia Tech, 338 Wallace Hall, Blacksburg, VA 24061; V. Kraak, PhD, RD; E. Serrano, PhD

Objective: This study examined compliance of foods and beverages available for purchase by students in vending machines and à la carte to evaluate compliance in rural Appalachian Virginia middle schools before and after implementation of the Smart Snacks in School federal regulation.

Design, Setting, and Participants: Audits of food and beverage products sold in vending machines and à la carte were completed in spring of 2014 and 2015 in eight middle 